\title{
Pandoraea apista Bacteremia in a COVID-Positive Man: A Rare Coinfection Case Report from North India
}

\author{
Sweta Singh ${ }^{1} \quad$ Chinmoy Sahu ${ }^{1, \odot}$ Sangram Singh Patel ${ }^{1} \quad$ Atul Garg $^{1} \quad$ Ujjala Ghoshal ${ }^{1}$ \\ ${ }^{1}$ Department of Microbiology, Sanjay Gandhi Post Graduate \\ Institute of Medical Sciences, Lucknow, Uttar Pradesh, India \\ Address for correspondence Chinmoy Sahu, MD (Microbiology) \\ Department of Microbiology, Sanjay Gandhi Post Graduate \\ Institute of Medical Sciences, Raebareli Road, Lucknow, Uttar \\ Pradesh 226014, India (e-mail: sahu.chinmoy@gmail.com).
}

\begin{abstract}
Pandoraea apista is a novel gram-negative bacillus usually isolated from respiratory specimens of cystic fibrosis patients. Few cases of bacteremia have also been reported due to this rare pathogen. Emergence of multidrug-resistant isolates of this bacillus is of grave concern. Here, we report a very interesting and unusual case of Pandoraea

Keywords

- Pandoraea apista

- bacillus

- cystic fibrosis

- bacteremia

- COVID

- pneumonia apista bacteremia in a coronavirus disease (COVID)-positive elderly diabetic man suffering from pneumonia. Prompt isolation and antibiotic sensitivity testing guided the patient's treatment and yielded favorable outcome. The need of automated methods for identification and sensitivity testing limits the reporting of this rare but important pathogen in hospital settings. Detailed research work and studies are needed in this direction to better understand this pathogen and its clinical manifestations for better patient outcome.
\end{abstract}

\section{Introduction}

Pandoraea species is a newly emerging multidrug-resistant pathogen usually isolated from cystic fibrosis (CF) patients. It was first discovered by Coenye et al in $2000^{1}$ as a gram-negative bacteria and was differentiated from other closely resembling pathogens like Burkholderia, Cupriavidus, and Ralstonia species. ${ }^{1}$ Literature search reveals few isolated case reports of this rare pathogen mostly from CF patients.,3 It has been isolated from a variety of clinical samples and also contributes to bacteremia cases in both $\mathrm{CF}$ as well as non-CF patients. However, the data of blood stream isolation of this pathogen is very scarce, due to either lack of identification or misidentification. These are sturdy organisms and are resistant to a variety of routinely used antibiotics. Here, we report a case of bacteremia caused by Pandoraea apista in a COVID-positive elderly man suffering from pneumonia.

\section{Case Report}

A 72-year-old man presented to the outpatient department of our hospital with chief complaints of breathlessness, dyspnea, and gastric upset with diarrhea lasting a week. He recalled the history of recent visit to his nephew's wedding in New Delhi, India, 13 days ago. On returning to his own city, he developed fever, sore throat, dyspnea, and gastric upset with upper respiratory tract symptoms that lasted 10 days. He was a known diabetic and was on insulin injections for the past 7 years. Due to the ongoing COVID pandemic, he was advised to get testing for COVID. The test turned out published online June 28, 2021
DOI https://doi.org/

$10.1055 / \mathrm{s}-0041-1730847$ ISSN $0974-2727$ (c) 2021. The Indian Association of Laboratory Physicians.

This is an open access article published by Thieme under the terms of the Creative Commons Attribution-NonDerivative-NonCommercial-License, permitting copying and reproduction so long as the original work is given appropriate credit. Contents may not be used for commercial purposes, or adapted, remixed, transformed or built upon. (https://creativecommons.org/licenses/by-nc-nd/4.0/).

Thieme Medical and Scientific Publishers Pvt. Ltd. A-12, 2nd Floor, Sector 2, Noida-201301 UP, India 
to be positive with reverse transcription-polymerase chain reaction (RT-PCR) and he was admitted in the isolation ward of our center. Treatment was initiated with injectable ambisone $150 \mathrm{mg}$ intravenous (IV) once a day (OD); aztreonam $1 \mathrm{~g}$ IV thrice a day; methylprednisolone $500 \mathrm{mg}$ IV OD; oral tablets like limcee and zincovit, and nebulization with duolin and budecort with random blood sugar monitoring. In spite of the ongoing treatment, his symptoms worsened over the next 2 days. Routine blood investigations revealed lymphopenia, and raised C-reactive protein (CRP) and procalcitonin levels. Radiological workup was planned on the same day and a high-resolution CT (HRCT) revealed ground-glass opacities all over the lung fields, especially in the lower zones ( - Fig. 1). A repeat COVID-19 PCR the next day was again positive and the patient was finally diagnosed as a case of COVID-19 pneumonia. Medications like hydroxychloroquine, favipiravir/remedesvir, and azithromycin were added to the treatment regimen. Over the next 4 days, the patient clinically deteriorated in spite of antiviral treatment. The $\mathrm{SPO}_{2}$ (oxygen saturation) was around $85 \%$ with room air and the patient was subsequently started on oxygen with nasal cannula. Various biomarkers including CRP, lactate dehydrogenase (LDH), D-dimers, and ferritin levels were increased significantly. The patient started to show signs of sepsis on the 12th day with acute respiratory distress syndrome (ARDS) and, hence, blood culture was sent in a pair of bactenecin (BACTEC) bottles (1 aerobic, 1 anaerobic) to the microbiology laboratory for further processing. His blood BACTEC culture bottle flagged positive after 24 hours and direct gram stain revealed multiple gram-negative bacilli ( - Fig. 2), which were then subcultured on blood and MacConkey agar plates (-Fig. 3). Biochemical tests were performed and the organism was weakly catalase positive, non-lactose fermenter, o-nitrophenyl- $\beta$-D-galactopyranoside (ONPG)negative and nitrate not reduced. Finally, matrix assisted laser desorption ionization time of flight mass spectrometry (MALDI-TOF MS) was done for confirmation and the final identification was done as Pandoraea apista from both the

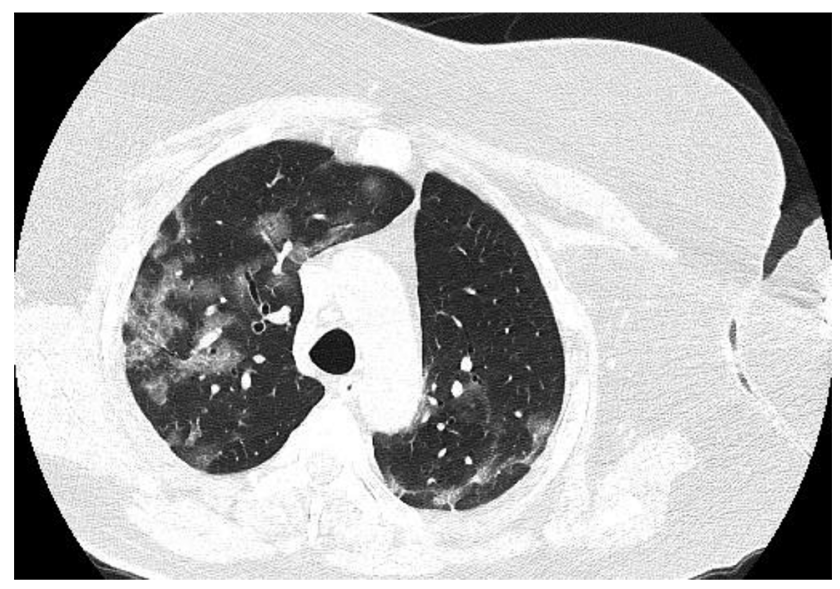

Fig. 1 High-resolution CT (HRCT) lung showing ground glass opacities in the coronavirus disease (COVID)-positive patient.

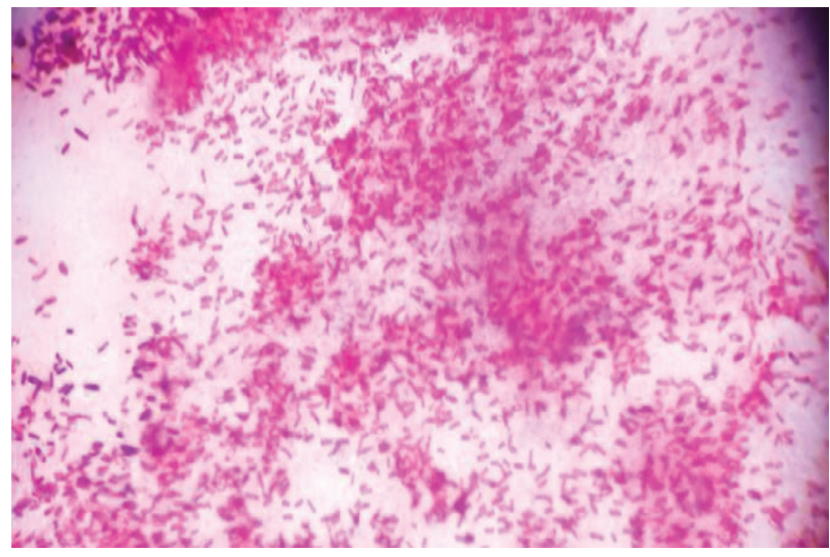

Fig. 2 Gram stain showing gram-negative bacilli of Pandoraea apista.
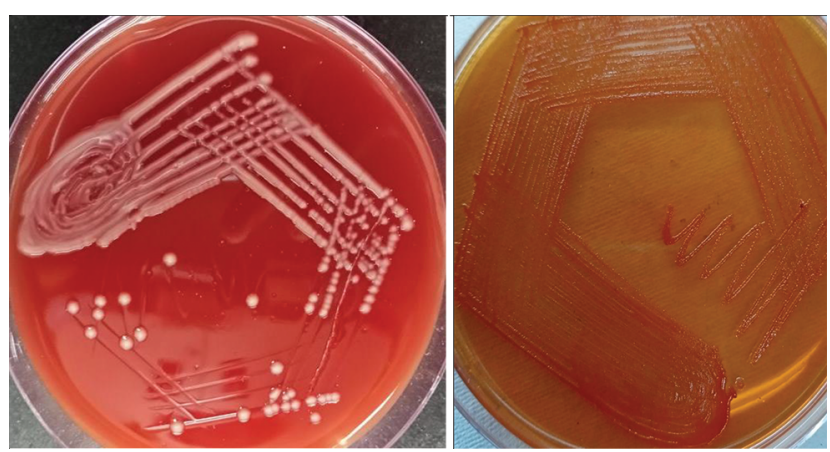

Fig. 3 Blood agar and MacConkey agar plates showing growth of Pandoraea apista.

aerobic and anaerobic bottles. Repeat blood cultures were ordered on the 16th day to confirm the isolate and prove its pathogenicity in sepsis in the patient. Pandoraea spp. was again isolated from the blood BACTEC bottles and its role was established in the clinical condition of the present case. Antibiotic sensitivity testing as per the recent clinical and laboratory standards institute (CLSI) guidelines revealed the organism to be sensitive to imipenem, minocycline, doxycycline, and trimethoprim-sulphamethoxazole, and resistant to ceftazidime, meropenem, tazobactam-piperacillin, colistin, cefoperazone-sulbactam, and amikacin. Imipenem was added to the treatment regimen as per recent sensitivity testing at a dose of $1 \mathrm{~g}$ IV, 6 hourly, along with tocilizumab infusion. In the next 3 days, there was improvement in oxygen saturation and temperature. Repeat blood tests revealed slight improvement in biomarkers and blood counts. Repeat RT-PCR for COVID-19 on the 16th day was finally negative and the computed tomography scans also showed improvement in the lung fields. Mechanical ventilation was avoided and the patient was continuously monitored for the next 7 days. His oxygen saturation improved and gradual resolution of symptoms was seen. Repeated blood culture during a follow-up revealed sterile results and the patient was able to maintain $92 \%$ oxygen saturation on room air. Subsequently, he was discharged on the 22nd day with advice for outpatient visits and routine blood investigation reports. 


\section{Discussion}

The COVID pandemic has led to the emergence of various coinfections in the form of bacterial and fungal colonizations in hospitalized patients. A case series from Washington DC, United States, reported bacterial coinfection rate as $4.8 \%$, while some studies from China also reported a rate of 5 to $9 \%{ }^{4}$ Gram-negative bacilli form the majority of this coinfection group.

Pandoraea species is a novel emerging pathogen group belonging to the subclass of Proteobacteria. They are gram-negative bacilli, aerobes or facultative anaerobes, nonsporing, non-lactose fermenters, ONPG-negative, and motile through flagella. ${ }^{1,2}$ The species which have been isolated from time to time from various human clinical specimens include P. apista, P. pulmonicola, P. pnomenusa, P. sputorum, and $P$. norimbergensis. ${ }^{1}$ Majority of the species were mainly isolated from CF patients as per previous literature case reports. ${ }^{2,3,5}$ Pandoraea is an environmental contaminant and is usually present in the soil and water. ${ }^{5}$

Various geographical areas, including Europe, Australia, and China, have reported the occurrence of Pandoraea in various clinical specimens. ${ }^{6,7}$ Most cases in the past have mostly and exclusively been reported from developed countries due to the complexity of isolation and identification of this rare species. Identification through routine biochemical tests and methods generally is not possible and may lead to misidentification and wrong reporting of the isolated species. ${ }^{7}$ This species can been wrongly identified as Ralstonia or Burkholderia species in the routine diagnostic laboratories. MALDI-TOF MS is a new automated system involved in rapid and accurate identification as well as antibiotic sensitivity testing of the isolates. ${ }^{1,2}$ It has revolutionized the world of microbiology by its varied merits in the patient's reporting as well as identification of the rare environmental pathogens which can have potential pathogenic roles.

The role of Pandoraea spp. in bacteremia is not well studied and very few cases have been reported from such patients. Past literature search reveals one such case of bacteremia in a lung transplant patient and another in an infant with acute lymphoblastic leukaemia. ${ }^{8,9}$ Coinfection can also be seen in such bacteremia cases along with the Pandoraea species as seen in past two cases. ${ }^{8,9}$ Pandoraea sputorum, P. pulmonicola, and $P$. apista have been reported and isolated from nonrespiratory samples too. ${ }^{1,5}$ Pandoraea apista is a novel pathogen causing bacteremia in hospitalized patients. The present case of $P$. apista in a COVID-positive patient emphasizes its role in sepsis. In the past also, its pathogenic role has been studied by some isolated case studies done by Stryjewski et al, in $2003,{ }^{8}$ and Johnson et al, in $2004 .{ }^{10}$

\section{Conclusion}

This case report highlights the important pathogenic role of this emerging multidrug-resistant pathogen in today's hospital settings. Automated methods like MALDI-TOF MS have helped a lot in timely and accurate diagnosis of this difficult-to-identify isolate. Our present case report is the first to identify the role of Pandorae aapista in sepsis in this part of the world, that is, North India. Still, further studies and research is required in this particular field to understand the pathogenesis and clinical implications of this rare pathogen on treatment and outcomes.

\section{Ethical Considerations}

Informed consent was obtained from the patient regarding the publication of images and clinical information in the journal. The patient was informed of the confidentiality of the data; however, the anonymity cannot be guaranteed.

\section{Author's Contribution}

S.S. and C.S. performed literature search and data analysis, and prepared the first draft of the manuscript and figures. S.S.P., A.G., and U.G. contributed in the final draft of the manuscript and editing.

\section{Research Quality and Ethics Statement}

The authors declare that this scientific work complies with reporting quality, formatting, and reproducibility guidelines set forth by the EQUATOR Network. We also certify that we have not plagiarized the contents in this submission and have done a plagiarism check.

\section{Conflicts of Interest}

None declared.

\section{References}

1 Coenye T, Falsen E, Hoste B, et al. Description of Pandoraea gen. nov. with Pandorae aapista sp. nov., Pandoraea pulmonicola sp. nov., Pandoraea pnomenusa sp. nov., Pandoraea sputorum sp. nov. and Pandoraea norimbergensis comb. nov. Int J SystEvolMicrobiol 2000;50(Pt 2) :887-899

2 Ambrose M, Malley RC, Warren SJ, et al. Pandoraea pnomenusa isolated from an Australian patient with cystic fibrosis. Front Microbiol 2016;7:692

3 Jeong SE, Lee HJ, Jia B, Jeon CO. Pandoraea terrae sp. nov., isolated from forest soil, and emended description of the genus Pandoraea Coenye et al. 2000. Int J SystEvolMicrobiol 2016;66(9):3524-3530

4 Feng Y, Ling Y, Bai T, et al. COVID-19 with different severities: a multicenter study of clinical features. Am J RespirCrit Care Med 2020;201(11):1380-1388

5 Daneshvar MI, Hollis DG, Steigerwalt AG, et al. Assignment of CDC weak oxidizer group 2 (WO-2) to the genus Pandoraea and characterization of three new Pandoraea genomospecies. J ClinMicrobiol 2001;39(5):1819-1826

6 Elborn JS. Cystic fibrosis. Lancet 2016;388(10059):2519-2531

7 Aravena-Román M. Cellular fatty acid-deficient Pandoraea isolated from a patient with cystic fibrosis. J Med Microbiol 2008;57(Pt 2) :252

8 Stryjewski ME, LiPuma JJ, Messier RH Jr, Reller LB, Alexander BD. Sepsis, multiple organ failure, and death due to Pandoraea pnomenusa infection after lung transplantation. J ClinMicrobiol 2003;41(5):2255-2257

9 Falces-Romero I, Gutiérrez-Arroyo A, Romero-Gómez MP. Catheter-associated bacteremia by Pandoraea pnomenusa in an infant with acute lymphoblastic leukemia. Med Clin (English edition) 2016;147(3):1320

10 Johnson LN, Han JY, Moskowitz SM, Burns JL, Qin X, Englund JA. Pandoraea bacteremia in a cystic fibrosis patient with associated systemic illness. Pediatr Infect Dis J 2004;23(9):881-882 